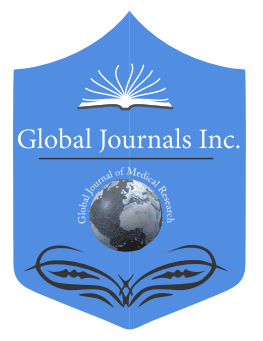

\title{
Urine Specific Gravity in Canine: Whole or Supernatant Sample?
}

\author{
By Laynez-Herrera, Olinda Maricruz, Villatoro-Chacón, Daniela Mariel, \\ Arizandieta-Altán, Carmen Grizelda, Chávez-López, Juan José \& Lepe-López, \\ Manuel Antonio \\ San Carlos of Guatemala University
}

Abstract- The ability to concentrate and dilute urine is one of the first functions that are lost as a result of tubular damage. Specific gravity by refractometry is a method used to evaluate this function. Some authors mention that the reactive sediment, as well as the presence of large amounts of high molecular weight substances can give an overestimation of the value of the specific gravity and recommends centrifuging the sample to obtain a reliable value. In the study, 123 urine samples and their specific gravity were evaluated before and after centrifugation. This in order to evaluate if the presence of reactive sediment, glucose and protein affected the value of the density and to obtain a reliable estimate in clinical practice. No significant difference was found between pre and post-centrifugation specific gravity measurement with portable refractometer $(W=8058.5, p$-value $=0.3759)$.

Keywords: specific gravity, refractometer, dogs.

GJMR-G Classification: NLMC Code: WA 360

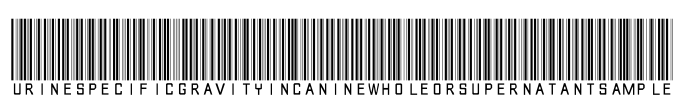

Strictly as per the compliance and regulations of:

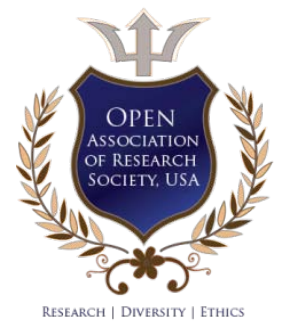

(c) 2019. Laynez-Herrera, Olinda Maricruz, Villatoro-Chacón, Daniela Mariel, Arizandieta-Altán, Carmen Grizelda, Chávez-López, Juan José \& Lepe-López, Manuel Antonio. This is a research/review paper, distributed under the terms of the Creative Commons Attribution-Noncommercial 3.0 Unported License http://creativecommons.org/licenses/by-nc/3.0/), permitting all non-commercial use, distribution, and reproduction in any medium, provided the original work is properly cited. 


\title{
Urine Specific Gravity in Canine: Whole or Supernatant Sample?
}

\author{
Densidad Urinaria En Caninos: ¿Muestra Entera O Sobrenadante?
}

\author{
Laynez-Herrera, Olinda Maricruz ${ }^{\alpha}$, Villatoro-Chacón, Daniela Mariel ${ }^{\circ}$, Arizandieta-Altán, Carmen Grizelda ${ }^{\circ}$, \\ Chávez-López, Juan José ${ }^{\omega}$ \& Lepe-López, Manuel Antonio ${ }^{*}$
}

Abstract- The ability to concentrate and dilute urine is one of the first functions that are lost as a result of tubular damage. Specific gravity by refractometry is a method used to evaluate this function. Some authors mention that the reactive sediment, as well as the presence of large amounts of high molecular weight substances can give an overestimation of the value of the specific gravity and recommends centrifuging the sample to obtain a reliable value. In the study, 123 urine samples and their specific gravity were evaluated before and after centrifugation. This in order to evaluate if the presence of reactive sediment, glucose and protein affected the value of the density and to obtain a reliable estimate in clinical practice. No significant difference was found between pre and postcentrifugation specific gravity measurement with portable refractometer $(W=8058.5$, $p$-value $=0.3759)$. However, since the interpretation of specific gravity is a categorical variable, 8 of 123 samples evaluated showed changes but only one change in their categorization. The findings found are important for the clinical assessment of outpatients or when a small amount of sample is available, allowing refractometry to be a reliable method without the need to centrifuge the urine to obtain the parameter.

Keywords: specific gravity, refractometer, dogs.

Resumen- La capacidad de concentrar y diluir la orina es una de las primeras funciones que se pierden como consecuencia del daño tubular. La densidad urinaria por refractometría es un método que se utiliza para evaluar esta función. Algunos autores mencionan que el sedimento reactivo, así como la presencia de grandes cantidades de sustancias de alto peso molecular pueden dar una sobreestimación del valor de la densidad y recomienda centrifugar la muestra para obtener un valor confiable. En el estudio, se evaluaron 123 muestras de orina y su densidad antes y después de centrifugar. Esto con el fin de evaluar si la presencia de sedimento reactivo, glucosa y proteína afectaba el valor de la densidad y poder obtener una estimación confiable en práctica clínica. No se encontró diferencia significativa entre la medición de la densidad pre y post-centrifugación con refractómetro portátil $(\mathrm{W}=8058.5$, p-valué $=0.3759$ ). Sin embargo, como la interpretación de la densidad urinaria es una variable categórica 8 de 123

Author $\alpha$ o $\rho$ : Departamento de Ayudas Diagnósticas, Hospital Veterinario, Facultad de Medicina Veterinaria y Zootecnia, Universidad de San Carlos de Guatemala, ciudad. e-mail: danavilla47@gmail.com Author w: Departamento de Clínicas de Animales de Compañáa, Hospital Veterinario, Facultad de Medicina Veterinaria y Zootecnia, Universidad de San Carlos de Guatemala, ciudad.

Author ¥: Programa en Medicina de Conservación, Facultad de Ciencias de la Vida, Universidad Andrés Bello, República 252, Santiago, Chile. muestras evaluadas mostraron cambios de densidad, pero solo una cambio su categorización. Los hallazgos encontrados, son importantes para la valoración clínica de pacientes ambulatorios o cuando se dispone de poca cantidad de muestra, permitiendo que la refractometría sea un método confiable sin necesidad de centrifugar la orina para la obtención del parámetro.

Palabras clave: densidad urinaria, refractómetro, perros.

\section{INTRODUCCIÓN}

a capacidad de los riñones para reabsorber en forma selectiva las sustancias químicas y agua a través del filtrado glomerular es una de las funciones más importantes del organismo al igual que la secreción de metabolitos y desechos (Strasinger y $\mathrm{Di}$ Lorenzo, 2010; Costa et al, 2010 y Bouda et al, 2007). La capacidad de concentrar y diluir la orina es una de las primeras funciones que se pierden como consecuencia del daño tubular, por consiguiente, la evaluación de esta capacidad es necesaria para el análisis de orina habitual. Esta evaluación se puede realizar mediante la determinación de la densidad urinaria, la cual también brindara información importante sobre el estado de hidratación del paciente (Strasinger y Di Lorenzo, 2010; Costa et al, 2010, Terres y González, 1992; Archer, 2012 y Campuzano y Arbeláez, 2007).

La densidad urinaria (o peso específico) es la relación entre el peso de un volumen dado de orina y el peso del mismo volumen de agua destilada (Costa et al, 2010 y Cortadellas y Fernández, 2012). Depende no solo del número de partículas que hay en solución, sino también de su peso molecular y de la temperatura (Calabia y Arias, 2008).

Se reportan tres métodos para su medición: refractómetro, urodensímetro y tiras reactivas, siendo este último no confiable a diferencia del refractómetro y el urodensímetro (Costa et al, 2010; Archer, 2012 y Cortadellas y Fernández, 2012).

El refractómetro portátil determina la densidad de manera accesible, rápida, fácil y con un volumen de muestra pequeña (Strasinger y Di Lorenzo, 2010). Este instrumento mide el grado de refracción de la luz por parte de los componentes sólidos cuando esta pasa a través de la orina (Bouda et al, 2007 y Archer 2012). El 
grado en que se doble el haz de luz es directamente proporcional al número de partículas disueltas en la solución (Wamsley y Alleman, 2007 y Archer, 2012). Sustancias que contribuyen al peso específico de la orina normal son: la urea (20\%), el cloruro de sodio (25\%), los sulfatos y los fosfatos (Fuller et al, 2005 y Riley y McPherson, 2017). Sin embargo, Wamsley y Alleman (2007), Terres y González (1992) e Idrovo (2018) mencionan que el sedimento reactivo (cristales, células, moco y bacterias) puede dar una sobreestimación de la densidad urinaria debido a que estos materiales también pueden desdoblar el haz de luz incrementando el valor de la densidad. Por esta razón, se recomienda hacer una evaluación de la densidad urinaria utilizando el sobrenadante de la muestra post centrifugación para obtener una estimación real de la capacidad tubular renal. Por otra parte, Calabia y Arias (2008) y Archer (2012) mencionan que se puede dar una sobreestimación de la densidad urinaria cuand o la muestra contiene grandes cantidades de glucosa, proteínas, lípidos y material de contraste.

En el presente estudio, se evaluaron 123 muestras de orina y su densidad antes y después de centrifugar. Esto con el fin de determinar si existe diferencia significativa en cuanto al valor de densidad y si la presencia de otros solutos afecta a este parámetro.

\section{il. Materiales y Métodos}

Se realizó el estudio en el Laboratorio Clínico del Hospital Veterinario de la Universidad de San Carlos de Guatemala, cuyas coordenadas son $14^{\circ} 34^{\prime} 58.44^{\prime} \mathrm{N}$ y $90^{\circ} 33^{\prime} 10.44 \mathrm{~W}$.

Se colectaron muestras de orina de 123 pacientes caninos que acudieron al Hospital Veterinario durante los meses de abril a julio de 2017; siendo estos de distintas razas, edades, sexo y tipo de recolección de muestra (chorro medio, sondaje, cistocentesis o del ambiente).

Se realizó examen completo a todas las muestras de orina colectadas, el cual consta de: Examen físico (color, olor, aspecto y densidad); examen químico ( $\mathrm{pH}$, proteínas, glucosa, cetonas, glucosa, bilirrubina, cetonas, urobilinógeno, sangre y leucocitos); y examen de sedimento (leucocitos, eritrocitos, células epiteliales, cilindros, cristales, bacterias, levaduras y parásitos). Sin embargo, las variables a considerar en el estudio fueron: Densidad, glucosuria, proteinuria con tira reactiva y prueba de Heller y presencia de sedimento reactivo (más de 6 elementos orgánicos o inorgánicos por campo: leucocitos, eritrocitos, células epiteliales, cilindros, cristales, bacterias y levaduras).

Para evaluar la densidad urinaria de la muestra completa y del sobrenadante se utilizó un refractómetro portátil marca Eurolab ${ }^{\circledR}$ previamente calibrado según la técnica descrita por Strasinger y Di Lorenzo (2010).

Para evaluar glucosuria se utilizaron tiras reactivas comerciales $\left(\right.$ Roche $\left.^{\circledR}\right)$. La glucosuria se midió a través de una escala de variación de color. Las tiras se colocaron en la orina homogenizada y sin centrifugar a temperatura ambiente y su lectura se realizó 45 a 60 segundos después de sumergidas comparando el cambio de color con la escala cromática provista por el fabricante. La lectura fue realizada visualmente.

Para la evaluación inicial de proteinuria se utilizaron tiras reactivas comerciales (Roche ${ }^{\circledR}$ ) debido a que representan el método más usado en medicina veterinaria por su valor económico y facilidad de uso (Beristain et al, 2010). Las tiras son sensibles particularmente a albumina (Cortadellas y Fernández, 2012). Por esta razón se utilizó la prueba de Heller (ácido nítrico al 65\%) para confirmar proteinuria (Hutter, 2010).

La evaluación de sedimento reactivo se realizó a través de la observación directa al microscopio binocular, se preparó la muestra como lo describen Diaz (1997) y Bouda, et al (2007).

Se utilizó el programa estadístico de libre acceso R versión 3.3.3 para el análisis de datos. El Test de Shapiro-Wilk expuso que los datos de densidad carecían de normalidad por lo que se utilizó la prueba no paramétrica de Wilcoxon.

\section{ili. Resultados}

Las 123 muestras evaluadas fueron recolectadas con distintos métodos. En el cuadro 1 se describen el número de muestras según el método de recolección.

Cuadro 1: Método de recolección de muestra.

\begin{tabular}{|c|c|c|}
\hline Método de recolección de muestra & No. De muestras & $\%$ \\
\hline Chorro medio & 61 & $49.59 \%$ \\
\hline Sonda uretral & 37 & $30.08 \%$ \\
\hline Cistocentesis & 13 & $10.57 \%$ \\
\hline Del Ambiente & 12 & $9.76 \%$ \\
\hline
\end{tabular}

En cuanto al sexo, más de la mitad de las muestras fueron de pacientes machos. En el cuadro 2 se presentan la distribución de las muestras según el sexo. 
Cuadro 2: Distribución de la población según sexo.

\begin{tabular}{|c|c|c|}
\hline Sexo & No. De muestras & $\%$ \\
\hline Machos & 64 & $52.03 \%$ \\
\hline Hembras & 59 & $47.97 \%$ \\
\hline
\end{tabular}

El rango de edad de los pacientes fue de $0.5 \mathrm{a}$ 19 años, con un promedio de $7.24 \pm 0.38$. Según la variable "edad" el primer cuartil agrupó a los individuos menores de 1 año y el tercer cuartil reunió ejemplares mayores de 16 años.

Respecto a la raza, los pacientes sin raza definida (SRD) fueron los pacientes con mayor frecuencia, seguido de los pacientes con raza definida como Poodle, Schnauzer y Shih tzu que obtuvieron

Cuadro 3: Distribución de la población según raza.

\begin{tabular}{|c|c|c|}
\hline Raza & No. de muestras & $\%$ \\
\hline SRD & 24 & $19.51 \%$ \\
\hline Poodle & 23 & $18.70 \%$ \\
\hline Schnauzer & 12 & $9.76 \%$ \\
\hline Shih tzu & 7 & $5.69 \%$ \\
\hline Otras razas & 57 & $46.34 \%$ \\
\hline
\end{tabular}

Respecto a la glucosuria sólo se ob servó en una pequeña proporción de la población (cuadro 4).

Cuadro 4: Distribución de pacientes con glucosuria

\begin{tabular}{|c|c|c|}
\hline Glucosuria & No, de muestras & $\%$ \\
\hline Sin glucosuria & 116 & $94.31 \%$ \\
\hline Con glucosuria & 7 & $5.69 \%$ \\
\hline
\end{tabular}

La proteinuria fue evaluada con tira reactiva y prueba de Heller. En el cuadro 5 se presentan las proporciones según técnica utilizada.

Cuadro 5: Evaluación de proteinuria por técnica

\begin{tabular}{|c|c|c|c|c|}
\hline & \multicolumn{3}{|c|}{ Técnica } \\
\hline & \multicolumn{2}{|c|}{ Tira reactiva } & \multicolumn{2}{c|}{ Reacción de Héller } \\
\hline Proteinuria & No. de muestra & $\%$ & No. de muestra & $\%$ \\
\hline Sin proteinuria & 49 & $39.84 \%$ & 58 & $47.15 \%$ \\
\hline$+(30 \mathrm{mg} / \mathrm{dl})$ & 66 & $53.66 \%$ & 65 & $52.85 \%$ \\
\hline$++(100 \mathrm{mg} / \mathrm{dl})$ & 8 & $6.50 \%$ & 0 & $0 \%$ \\
\hline$+++(1000 \mathrm{mg} / \mathrm{dl})$ & 0 & $0 \%$ & 0 & $0 \%$ \\
\hline$++++(>1000 \mathrm{mg} / \mathrm{dl})$ & 0 & $0 \%$ & 0 & $0 \%$ \\
\hline
\end{tabular}
(cuadro 6).

En cuanto a la evaluación de sedimento reactivo, más de la mitad de la población fue positivo al mismo

Cuadro 6: Presencia de sedimento

\begin{tabular}{|c|c|c|}
\hline Sedimento & No. de muestra & $\%$ \\
\hline Con sedimento & 83 & $67.48 \%$ \\
\hline Sin sedimento & 40 & $32.52 \%$ \\
\hline
\end{tabular}

Se realizó la categorización clínica de la densidad urinaria de las muestras evaluadas según los criterios de Wamsley y Alleman (2007). La mayor proporción de los pacientes tanto pre y post centrifugación presento orina bien concentrada (cuadro 7, figura 1). mayores frecuencias (cuadro 3). Otras razas que se Chow, Cocker, Husky Siberiano, Chihuahua, Rottweiler, Akita, Alaskan Malamute, Dachshund, Pitbull, American Eskimo, Boston Terrier, Bulldog Inglés, Dálmata, Doberman, Pinscher, Dogo, Lhasa Apso, Mastín Inglés, Viejo Pastor Inglés y West Highland White Terrier. 
Cuadro 7: Valorización y categorización clínica de la densidad urinaria pre y post centrifugación del total de la población.

\begin{tabular}{|l|c|c|c|c|}
\hline & \multicolumn{2}{|c|}{ Pre - centrifugación } & \multicolumn{2}{c|}{ Post - centrifugación } \\
\hline \multicolumn{1}{|c|}{ Densidad } & No. de muestras & $\%$ & No. de muestras & $\%$ \\
\hline Hipostenuria (<1.008) & 12 & $9.76 \%$ & 12 & $9.76 \%$ \\
\hline Isostenuria (1.008-1.012) & 9 & $7.32 \%$ & 9 & $7.32 \%$ \\
\hline Concentración Mínima (1.013-1.029) & 33 & $26.83 \%$ & 34 & $27.64 \%$ \\
\hline Bien concentrada (>1.030) & 69 & $56.09 \%$ & 68 & $55,28 \%$ \\
\hline
\end{tabular}

Densidad Pre centrifugación

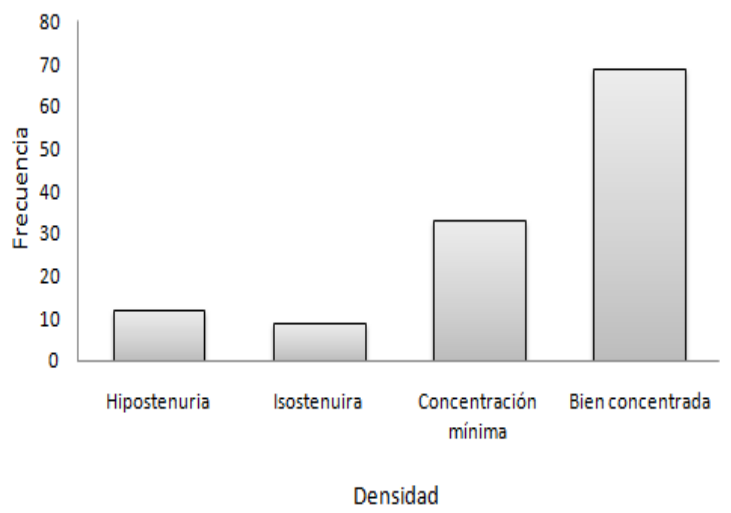

Densidad Post centrifugación

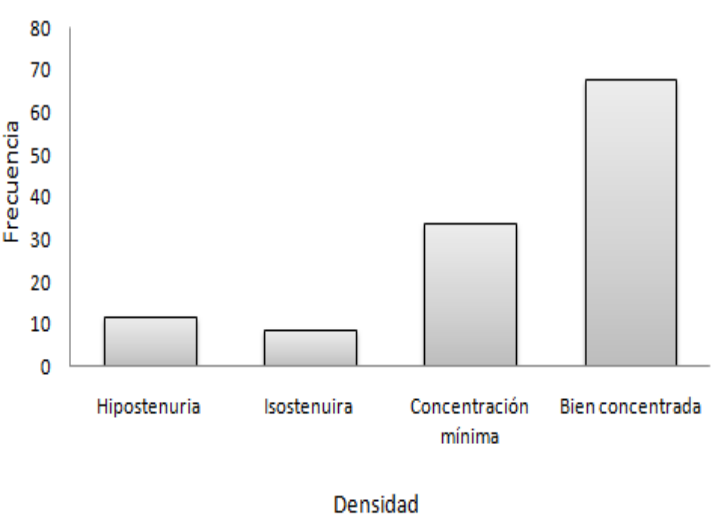

Figura 1: Valoración y categorización de la densidad pre y post centrifugación de la población

Se constató que no existe diferencia estadísticamente significativa entre la medición pre y post centrifugación de densidad urinaria $(\mathrm{W}=8058.5$, p-valué $=0.3759$ ). Debido a que no se encontró diferencia significativa en la densidad urinaria pre y post centrifugación, no fue posible evaluar el efecto del análisis químico y de sedimento sobre la densidad urinaria.

Sin embargo, ya que el significado de la densidad es categórico, se agruparon los resultados del análisis pre y post centrifugación en una tabla tipo pivote, verificando que únicamente en un individuo se alteró su estado de bien concentrada a concentración mínima después de la centrifugación

\section{Discusión}

Del total de la población el $52.03 \%$ fueron machos, siendo similar a lo reportado por Alvarado et al (2017). Esto sugiere que tanto machos como hembras son adquiridos de igual manera y la tenencia de hembras no es un problema al disponer de métodos que permiten el control de la fecundidad, como lo son las esterilizaciones quirúrgicas y tratamientos farmacológicos (Ibarra et al., 2003). Así mismo, los caninos de edad adulta fueron quienes presentaron mayor asistencia a consulta veterinaria.

En cuanto a raza, los caninos SRD fueron quienes obtuvieron mayor frecuencia en el estudio. Dentro de las causas puede considerarse el incremento descontrolado de la población canina, el crecimiento de población humana y el aumento de la tasa de migración de las personas del campo a la zona urbana que conlleva un traslado de animales domésticos (Lucio et al., 2017). Del mismo modo, el incremento en la concientización sobre la adopción de perros sin hogar (en su mayoría SRD) por parte de entidades públicas, empresas privadas y asociaciones no gubernamentales (Pacay, 2015). Se estima que en el mundo existen alrededor de 500,000,000 de perros, de los cuales el $75 \%$ son no poseen pedigree o no son cruces de dos razas conocidas (Cadena y Lenin, 2013). Por otra parte, la tenencia de razas como Poodle, Schnauzer y Shih tzu puede atribuirse al espacio físico, cuidado y alimentación que estas razas requieren por ser de talla pequeña-mediana (Alvarado et al., 2017).

Respecto a los métodos de recolección de muestra, el método más utilizado fue el chorro medio. Esto pudo deberse a que las muestras colectadas por chorro medio son las más habituales para obtener muestras de tamiz debido a la facilidad de recolección, comodidad para el paciente, ser un método seguro y menos traumático (Strasinger y Di Lorenzo, 2010 y Chew y DiBartola, 1998). Se recomienda que la muestra sea obtenida de la primera orina de la mañana ya que es concentrada y refleja mejor el estado general del paciente, siendo la parte media más representativa ya que la primera fracción puede arrastrar componente de la uretra o del tracto genital (Bouda et al., 2007; Diaz et al., 1997 y Chew y DiBartola, 1998). Por otra parte, las muestras obtenidas por sondaje o cistocentesis en la 
mayoría de las situaciones son solicitadas con fines específicos (cultivo bacteriano o citología) conllevan mayor pericia y mayores riesgos (Strasinger y Di Lorenzo, 2010). Las muestras tomadas del ambiente son menos apropiadas y por lo tanto fueron las menos frecuentes. Por ello, se debe recurrir a ellas cuando son la única opción para obtener la muestra considerando la contaminación de las mismas al momento de la interpretación de los resultados (Chew y DiBartola, 1998).

Se observó glucosuria en el 5.69\% de las muestras procesadas. Esta prevalencia se asemeja a lo reportado por Mesa y Castillo (2014) y Zamora y Osorio (Zamora et al., 2015). Estos datos sugieren lo poco frecuente de este hallazgo. Sin embargo, ya que la glucosuria está asociada a varias patologías y su origen puede ser renal o extra-renal, se recomienda realizar mediciones seriadas simultaneas de glicemia y glucosuria. Esto permitirá identificar el origen y por consiguiente obtener un diagnostico e implementar un tratamiento adecuado (Campuzano y Arbeláez, 2007 y Zamora et al., 2015).

El $60.16 \%$ de los pacientes presentaron proteinuria con el método de tira reactiva, mientras que sólo el 52.85\% fue reactivo con la prueba de Heller. Estos datos son similares a otros estudios sugiriendo que la proteinuria es un hallazgo frecuente en perros durante la consulta médica rutinaria (Beristain et al., Gallo y Silva, 2014 y Barros y Carpio, 2017). Sin embargo, la presencia de proteínas en orina no constituye una prueba de nefropatía ni su ausencia la excluye ${ }^{6}$. Por esta razón se debe determinar su origen ya que este suele ser variado y no siempre patológico (Campuzano y Arbeláez, 2007; Cortadellas y Fernández, 2012; Beristain et al., 2010; Zamora et al., 2015 y Barros y Carpio, 2017). Cabe mencionar que en condiciones normales en los perros existe una ligera proteinuria que oscila entre 0.1 a $0.3 \mathrm{~g} / \mathrm{l}$ (Barros y Carpio, 2017). Además, la proteinuria en trazas $01+$ en orina muy concentrada a menudo se atribuye a la concentración de la orina y no a una proteinuria anormal (Grauer, 2016). La eliminación urinaria de una cantidad excesiva de proteínas de forma persistente es un marcador de daño renal (Calabia y Arias, 2008).

El $67.48 \%$ de las muestras fueron positivas a sedimento reactivo. Este parámetro pudo estar relacionado al método de toma de muestra (Chew y DiBartola, 1998). Se debe considerar que el sedimento urinario de un individuo sano no está libre de elementos formes, pero contiene un número limitado de estos. Por esta razón, si se encuentran en muy pequeña proporción por campo no se consideran necesariamente anormales (Campuzano y Arbeláez, 2007; Calabia y Arias, 2008 y Díaz et al., 1997). Además, la primera orina de la mañana ofrece un mayor número de elementos celulares y bacterias, que generalmente provienen de uretra, vagina y prepucio.
Por este motivo, es de suma importancia una amplia experiencia para su análisis y una adecuada interpretación tomando en cuenta la condición clínica de cada paciente (Zamora et al., 2015).

La densidad que presentó mayor frecuencia fue $>1.030$. Esto nos sugiere que la mayoría de los caninos evaluados presento correcta capacidad de concentrar la orina. Se encontró que el $26.83 \%$ presento concentración mínima mostrando una alteración significativa en la capacidad de concentración renal. Sólo el $7.32 \%$ presentó isostenuria lo que indica que el riñón no concentra ni diluye adecuadamente la orina. Sin embargo, la evaluación de la densidad urinaria debe realizarse de forma discreta, ya que un perro sano puede diluir o concentrar la orina entre 1.001 a 1.075 (Watson et al., 2015). Por esta razón, el análisis de la densidad debe evaluarse según las circunstancias clínicas de cada paciente y colectar las muestras por la mañana antes que el animal haya ingerido agua o comida ya que estas muestras son más útiles en la evaluación de la concentración de la orina. De esta manera, se podrá obtener una correlación clínica y patológica de la capacidad de concentración renal (Chew y DiBartola, 1998). Además, debe considerarse que la densidad varía en el curso del día y que una sola lectura no da la información suficiente, por ello se recomienda realizar varias mediciones (Graff, 2007).

Por otra parte, no se encontró diferencia significativa entre la medición de la densidad pre y postcentrifugación con refractómetro portátil. El valor diagnóstico de este hallazgo nos permite realizar una estimación adecuada y confiable de la densidad en muestras sin centrifugar de pacientes ambulatorios con orinas normales o cuando se disponga de poca cantidad de orina (Terres y González, 1992 y Díaz et al., 1997). Dado que en el estudio no se observó diferencia significativa de la densidad urinaria pre y postcentrifugación, las variables proteinuria, glucosuria y sedimento no fueron evaluadas. Sin embargo, autores como Terres y González (1992), Strasinger y Di Lorenzo (2010) y Archer (2012) reportan que la densidad urinaria depende de la naturaleza y del número solutos, por lo que grandes cantidades de sustancias de alto peso molecular (albumina o glucosa) incrementan la densidad urinaria. Este factor da la falsa impresión de que el riñón es capaz de concentrar la orina. Por lo tanto, en estos casos se recomienda realizar una corrección numérica al resultado de la medición o medir directamente la osmolaridad para establecer la verdadera capacidad renal de concentrar y diluir la orina. Sin embargo, a pesar de que la osmolaridad es la medida de elección para evaluar la capacidad de concentración renal, en la práctica clínica, es un método poco accesible a diferencia de la densidad urinaria (Costa et al., 2010; Terres y González, 1992; Calabia y Arias, 2008 y Graff 2007). 


\section{Conclusion}

No se encontró diferencia significativa entre la medición de la densidad pre y post-centrifugación con refractómetro portátil. Por tal razón medir la densidad urinaria en una muestra de orina del sobrenadante o sin centrifugar no afecta el valor diagnóstico en los pacientes. Esto permite que este método brinde datos confiables para la valoración de los mismos en la práctica clínica.

\section{Literatura Citada}

1. Alvarado M, Villatoro D, Chávez J, Arizandieta C. Caracterización de la población canina atendida en el centro municipal de atención canina de la ciudad de Guatemala. Rev Electrón Vet. 2017; 18 (12): $1-9$.

2. Archer Joy. Análisis de Orina. En: Villiers E, Blackwood L. Eds. Manual de Diagnóstico de Laboratorio en pequeños animales. 2da edición. España: Lexus; 2012. 209-235.

3. Barros E, Carpio $F$ (dir). Efectividad de los métodos ácido sulfosalicilico, héller y tira reactiva para el diagnóstico temprano de proteinuria en perros que presentan cilindros en sedimento urinario. [Tesis de grado en internet]. Ecuador: Facultad de Ciencias Agropecuarias Carrera de Medicina Veterinaria, Universidad de Cuenta; 2017. 64p. [Citado 2 de mayo del 2018]. Disponible en: http://dspace. ucuenca.edu.ec/handle/123456789/28438

4. Beristain D, Barrera R, Rodríguez C. Duque J, Ruiz P, Zaragoza C. Causas de proteinuria post renal en el perro, estudio retrospectivo de 162 casos. Rev Electrón Vet [internet]. 2009. [citado 13 de febrero del 2018]; 10 (5): 1-7. disponible en http://www. veterinaria.org/revistas/redvet/n050509/050904.pdf

5. Beristain M, Zarag oza C, Duque P, Ruiz P, Barrera R. Manejo Laboratorial e Interpretación de la Proteinuria en el Perros, pasos a seguir. Rev AMVAC. [internet]. 2010 [Citado 13 de marzo del 2018]. 37: 4 -15. Disponible en: http://axonve terinaria.net/web axoncomunicacion/centroveterinar io/37/cv_37_Revista_completa.pdf

6. Bouda J, Doubek J, Quiroz G. Patología clínica del aparato urinario. En: Nuñez L, Bouda J. Eds. Patología clínica veterinaria. 2da edición. México: FMVZ-UNAM. 2007. p.99-119.

7. Calabia E, Arias M. Estudio de la Función Renal en: Hernando L, Aljama P, Arias M, Caramelo C, Egido J. Lamas S. Nefrología Clínica. 3era edición. España: Panamericana; 2008. p 135-143.

8. Calabia E, Arias M. Análisis de orina en: Hernando L, Aljama P, Arias M, Caramelo C, Egido J. Lamas S. Nefrología Clínica. 3era edición. España: Panamericana; 2008. p 127-135.

9. Campuzano G, Arbeláez M. E I Uroanálisis: Un gran aliado del médico. Rev Urol Colom [Internet].
2007 [Citado 11 de febrero del 2018]; 56 (1): 67-92. Disponible en: http://www.redalyc.org/articulo.oa? id $=149120468005$

10. Cadena $G$, Lenin V (dir). Estudio para la estimación de la población de perros callejeros en mercados municipales del distrito metropolitano de quito. DMQ. [Tesis de grado]. Quito: Colegio de Ciencias de la Salud, Universidad San Francisco de Quito; 2013. 139p.

11. Chew D, Di Bartola S. Interpretación del Uroanálisis Canino y Felino. EEUU, Ralston Purina Company. 1998. 43p.

12. Cortadellas $O$, Fernández $M$. Diagnóstico y tratamiento de la enfermedad renal crónica (ERC) en el perro y el gato. Parte 1: evaluación del paciente con ERC. Clin Vet Peq Anim [Internet]. 2012 [Citado 21 de febrero del 2018]; 32 (4): 215-223. Disponible en: https://ddd.uab.cat/pub/ artpub/2012/130278/clivetpeqaniv32n4p215.pdf

13. Costa E, Bettendorff C, Bupo S, Ayuso S, Vallejo G. Medición comparativa de la densidad urinaria: tira reactiva, refractómetro y densímetro. Arch Argent Pediatr. 2010; 108 (3): 234 -238.

14. Diaz J, Fernández M, Paredes F. Aspectos básicos de Bioquímica clínica. $1^{\mathrm{a}}$, Ed. Madrid: Ediciones Diaz de Santos; 1997. 304p.

15. Fuller C, Threatte G, Henrry J. Examen básico de la orina. En: Henry Jhon, Davey F, Herman C, McPherson R, Pincus M, Threatte G, Woods G. Eds. E I laboratorio en el diagnóstico clínico: homenaje a todd sanford \& davidsohn. Vol 1. 20a ed. España: Marbán; 2005. p. 367-402.

16. Gallo C, Silva M (dir). Manual de Diagnostico con Énfasis en Laboratorio Clínico Veterinario. [Trabajo final de grado]. Managua: Facultad de Ciencia Animal Carrera de Medicina Veterinaria, Universidad Nacional Agraria; 2014.211p.

17. Graff S. Análisis de Orina, Atlas color. 2da Edición. Mexico D.F.: Editorial medica panamericana; 2007. 222p.

18. Grauer G. Proteinuria [internet]. International Renal Interest Society IRIS. Manhattan, USA. 2016. [citado 2 de abril del 2018]. Disponible en: http://www.iriskidney.com/education/proteinuria.html

19. Hutter E. Analisis Rapido de Orina [Internet]. 2010 [citado 12 de febrero del 2018]. 73-79p. Disponible en: http://www.rednacionaldeveterinarias.com.uy/ articulos/laboratorio/Analisis_rapido_de_orina_2010. pdf

20. Ibarra L, Morales M, Acuña P. Aspectos demográficos de la población de perros y gatos en la ciudad de Santiago, Chile. Rev Av cienc vet [Internet]. 2003 [Citado 5 de febrero del 2018]; 8 $(1,2)$ : 14-20. Disponible en: http://repositorio.uchile. $\mathrm{cl} /$ handle/2250/122610

21. Idrovo Ortiz J, Méndez M (dir). comparación de dos protocolos anestésicos utilizados para ovariohi- 
sterectomía en perras y su impacto en la función renal mediante análisis sanguíneo y urianálisis. [Tesis de maestría en internet]. Ecuador: Faculta de Ciencias Agropecuarias Carrera de Medicina Veterinaria. Universidad de Cuenca; 2018. 65p [Citado 2 de marzo del 2018]. Disponible en: http://dspace.ucuenca.edu.ec/bitstream/123456789 /29513/1/Trabajo\%20de\%20Titulaci\%C3\%B3n.pdf

22. Lucio A, Yánez D, Donato J, Guillín F, Flores A, Sanchez D, Heras L, Valverde P. Sobrepoblación de perros callejeros y la transmisión de enfermedades zoonóticas a la población en el sector del mercado 10 de noviembre de la ciudad de Guaranda en el periodo de octubre 2016 - febrero 2017. En: IV Congreso Internacional de Ciencia y Tecnología, Innovación y Emprendimiento (CTIE): 5, 6 y 7 de julio del 2017 Ecuador: universidad Estatal de Bolívar; 2017. p. 293-297.

23. Mesa D, Castillo A, Contreras S (dir). Prevalencia de diabetes mellitus en caninos con edad mayor 0 igual a 5 años del barrio Juan Alberto Blandón del municipio de Estelí utilizando como método diagnostico el glucómetro ACON On call en el periodo comprendido de agosto a septiembre 2013. [Tesis de grado en Internet]. León: Escuela de Medicina Veterinaria, Universidad Nacional Autónoma de Nicaragua; 2014. 46p. [Citado 8 de abril del 2018]. Disponible en: http://riul.unanleon. edu.ni:8080/jspui/bitstream/123456789/3307/1/2280 63.pdf

24. Pacay M. Adopción de Mascotas. [noticia en blog de Internet]. Amiga. 3 de abril del 2015. [citado 18 de junio del 2018]. Disponible en: https://www. revistaamiga.com/actualidad/adopcion-mascotas/

25. Riley R, McPherson R. Basic examination of urine. En: McPherson R, Pincus M. editores. HENRY'S Clinical Diagnosis and Management by Laboratory Methods. 23rd ed. St. Louis, Mossouri (USA): Elsevier; 2017. p. 442-480.

26. Strasinger S, Di Lorenzo M. Análisis de orina y de los líquidos corporales. 5ta Edición. Buenos Aires: Panamericana; 2010. 320p.

27. Terres A, González R. Estimación de la osmolaridad urinaria en base a la densidad urinaria. Rev Mex Patol Clin [Internet]. 1992 [citado 11 de febrero del 2018]; 39 (1): 8-13. Disponible en: https://books. google.com.gt/books?id=SjhmIPLO4k0C\&pg =PA1 $0 \& d q=$ densidad + urinaria\&hl $=$ es $-419 \&$ sa $=X \&$ ved =0ahUKEwjpjc_StfLYAhXL2IMKHVGQCo8Q6AEIKZ $A B$

28. Wamsley $\mathrm{H}$, Alleman R. Complete urinalysis. En: Elliott J, Grauer G. editores. BSAVA manual of Canine and Feline Nephrology an Urology, 2da ed. England: BSAVA; 2007. 87-95.

29. Watson A, Lefebvre H, Elliott J. Urine specific gravity [Internet]. International Renal Interest Society IRIS. 2015. [fecha de acceso 16 de marzo del 2018].
Disponible en http://www.iris-kidney.com/education/ urine_specific_gravity.html

30. Zamora M, Osorio V, Peralta A (dir). Descripción de hallazgos clínicos en el examen general de orina en caninos con patología del tracto urinario atendidos en la Clínica Veterinaria UNAN-León en el periodo agosto - diciembre 2014. [Tesis de grado en internet]. León: Escuela de Medicina Veterinaria, Universidad Nacional Autónoma de Nicaragua; 2015. 72p. [Citado 27 de abril del 2018]. Disponible en: http://riul.unanleon .edu.ni:8080/jspui/ bitstream/ 123456789/4092/1/229273.pdf 